\title{
Influence of the Thin-Film Ag Electrode Deposition Thickness on the Current Characteristics of a CVD Diamond Radiation Detector
}

\author{
Chae-Min Ban*, Chul-Yong Lee, Byung-Hyuk Jun \\ Neutron Utilization Research Division, Korea Atomic Energy Research Institute, Daejeon, Korea
}

\section{Technical Paper}

Received June 28, 2018

Revision October 9, 2018

Accepted November 17, 2018

Corresponding author: Chae-Min Ban

Neutron Utilization Research Division, Korea Atomic Energy Research Institute, 989-111 Daedeok-daero, Yuseong-gu,

Daejeon 34057, Korea

Tel: $+82-42-868-2037$

Fax: +82-42-863-6522

E-mail:minni20@naver.com

This is an Open-Access article distributed under the terms of the Creative Commons Attribution NonCommercial License (http://creativecommons.org/ licenses/by-nc/4.0) which permits unrestricted noncommercial use, distribution, and reproduction in any medium, provided the original work is properly cited.

Copyright $\odot$ 2018 The Korean Association for Radiation Protection

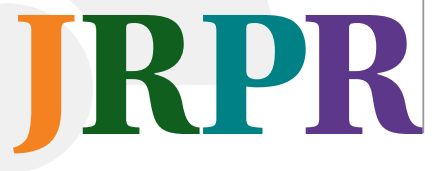

Background: We investigated the current characteristics of a thin-film Ag electrode on a chemical vapor deposition (CVD) diamond. The CVD diamond is widely recognized as a radiation detection material because of its high tolerance against high radiation, stable response to various dose rates, and good sensitivity. Additionally, thin-film Ag has been widely used as an electrode with high electrical conductivity.

Materials and Methods: Considering these properties, the thin-film Ag electrode was deposited onto CVD diamonds with varied deposition thicknesses ( $\fallingdotseq 50 / 98 / 152 / 257 \mathrm{~nm})$; subsequently, the surface thickness, surface roughness, leakage current, and photo-current were characterized.

Results and Discussion: The leakage current was found to be very low, and the photo-current output signal was observed as stable for a deposited film thickness of $98 \mathrm{~nm}$; at this thickness, a uniform and constant surface roughness of the deposited thin-film Ag electrode were obtained.

Conclusion: We found that a CVD diamond radiation detector with a thin-film Ag electrode deposition thickness close to $100 \mathrm{~nm}$ exhibited minimal leakage current and yielded a highly stable output signal.

Keywords: CVD diamond, Radiation detector, Thin-film Ag electrode, Leakage current, Photo-current

\section{Introduction}

Radiation is a flow of energy that has the ability to directly or indirectly ionize air from particle lines or electromagnetic waves. The types of radiation include alpha radiation, beta radiation, gamma radiation, neutron radiation, ultraviolet radiation, and X-rays. Beginning with Kozlov et al. [1] in 1966, the unique properties of diamond have been explored to detect and characterize radiation. At present, high-quality single crystal chemical vapor deposition (CVD) diamond is used as a radiation detector material because it is easily compacted, which facilitates ideal positioning, and exhibits resistance to radioactivity without generating a secondary fission [2].

CVD diamond has a wide bandgap of $5.5 \mathrm{eV}$, high resistivity, high thermal conductivity, high electron and hole mobilities, which enable faster charge collection, and radiation robustness [3]. Based on such properties, CVD diamond can be considered as an interesting candidate for use as a photodetector device that is able to detect extreme- to 
deep-UV radiation while also being transparent in the visible wavelength. Moreover, CVD diamond-based electronics can operate in high-temperature and harsh environments, e.g., under the condition of ionizing radiation, where the operation of conventional semiconductor devices is limited [4-8]. These unique characteristics have been realized in devices capable of replacing silicon-based devices, such as thermal and fast neutron detectors in nuclear fusion reactors [9]. Radiation detectors are also required in such applications, and CVD diamond-based devices can offer excellent performance with great optical efficiency and robustness in extreme environments.

The thin-film electrode deposition of a CVD diamond radiation detector is the process of electrically connecting each element formed through various processes in order to complete the circuit of the detector [10]. Ag is used as the thinfilm electrode of a CVD diamond radiation detector because it possesses high electrical conductivity and the ability to establish good ohmic contact with low resistivity. Furthermore, since the conditions for hole and electron injections are better than those for other metals, the charge mobility is improved. In addition, when the bias voltage is increased, the leakage current remains constant and stable [11]. In order to confirm this, we conducted a comparison between the current leaked due to an increase in the bias voltage of the deposited Ag and that of Pt metal on CVD diamond. For example, to prepare good injecting contacts, several metals were used by Kozlov et al ${ }^{1}$., for hole injection they used Ag, Au, Pt, or $\mathrm{C}$ deposition, aluminium or boron implantation, for electron injection $\mathrm{P}, \mathrm{Li}$, or C. Consequently, it was found that the deposited Ag sample had a lower leakage current than the Pt sample that steadily increased with increasing bias voltage. The results also demonstrated that Ag metallization is more suitable for a CVD diamond radiation detector than any other metal electrode. Additionally, thin-film Ag electrodes have been reported to retain high electrical conductivity, and have good charge mobility and low resistivity even with a film thickness below $100 \mathrm{~nm}[12,13]$.

In this study, the following experiment and analysis were carried out as based on the electrical characteristics of the $\mathrm{Ag}$ electrode metallized to a thickness of $100 \mathrm{~nm}$, as was mentioned above. Leakage current changes under a bias voltage, and the photo-current under UV irradiation, were measured for various film thicknesses due to different thin-film Ag electrode deposition times. We also analyzed the surface and current characteristics of the deposited thin-film Ag electrode to achieve a highly efficient and sensitive CVD diamond radiation detector [14].

\section{Materials and Methods}

The photodetector device used in this study is an Element Six product, which is a single-crystal high-purity CVD diamond with dimensions of $3.0 \times 3.0 \times 0.5 \mathrm{~mm}^{3}$. Ag was deposited onto the CVD diamond substrate by performing $\mathrm{AC} \mathrm{RF}$ sputtering for durations of 20,40,60, and 80 seconds at 100 W. Silver paste was used to connect the MI (mineral-insulated) cable for the signal line to the deposited thin-film Ag electrode on the CVD diamond, as is shown in Figure 1. The MI cable is a mineral-insulated cable that demonstrates excellent long-term stability and enables high precision at high temperatures. Because of this characteristic, the MI cable
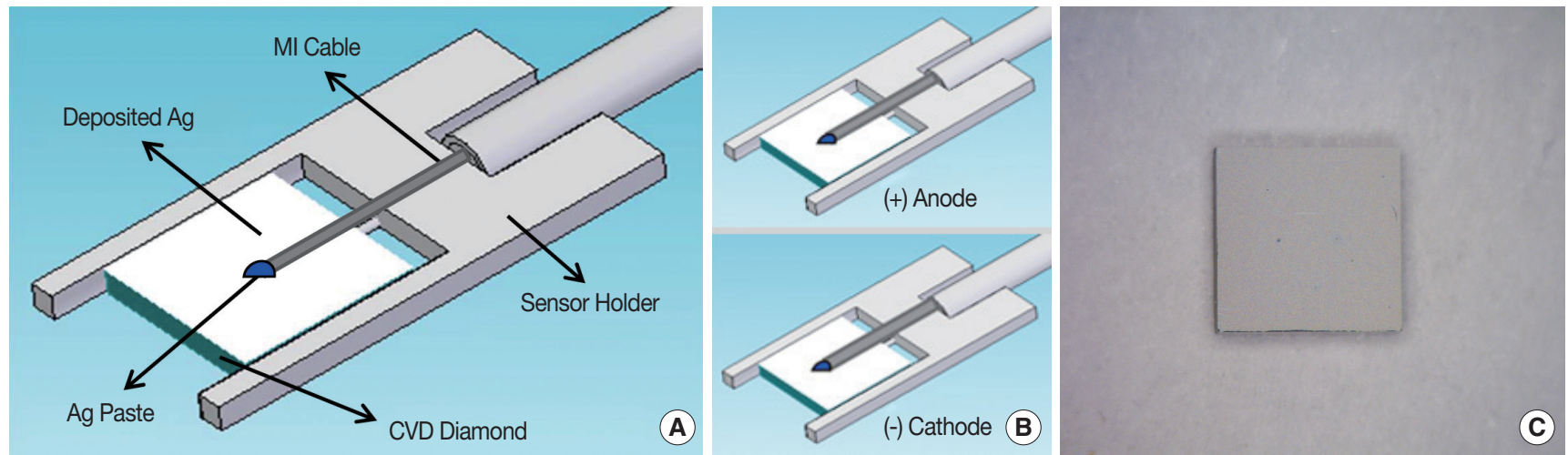

Fig. 1. Diagram of Ml cable connection to deposited thin-film Ag electrode on CVD diamond (A); cathode and anode side of deposited thinfilm Ag electrode (B); picture of deposited thin-film Ag electrode on CVD Diamond (C).

1) Kozlov SF, Konorova EA. Nuclear radiation detector and method for manufacturing same. U.K. Patent, G.B. 1970;1205115. 
can be used in extreme environments, such as in a nuclear reactor. The CVD radiation detector fabricated from this material was placed in a fixed jig to ensure that accurate leakage and photo-current measurements were obtained.

To monitor the current characteristics, the MI cable was connected to measure the leakage current and photo-current with the Keithley 6487 pico-ammeter (Tektronix, Inc., Beaverton, OR). The leakage current measurement was performed under the conditions of an applied 50-V bias [15, 16], and the photo-current was measured under 254-nm UV irradiation. This specific wavelength was chosen because of the characteristic response of the CVD diamond within a wavelength range of 200 to $300 \mathrm{~nm}[17,18]$.

To study the thickness and surface roughness of the de- posited thin-film Ag electrode, the thin-film Ag electrode was deposited onto a glass substrate over a period of 20,40, 60 , or 80 seconds, and was analyzed by using a scanning electron microscope (SEM) to confirm the cross-sectional thickness of the deposited electrode. Also, we analyzed the surface roughness of the electrode deposited onto the CVD diamond by using an atomic force microscope (AFM).

\section{Results and Discussion}

The cross-sectional thicknesses of the 20-, 40-, 60-, and 80-second deposited thin-film Ag electrodes were approximately 50, 98, 152, and $257 \mathrm{~nm}$, respectively, as is shown in Figure 2. Note that the deposited thin-film thickness values
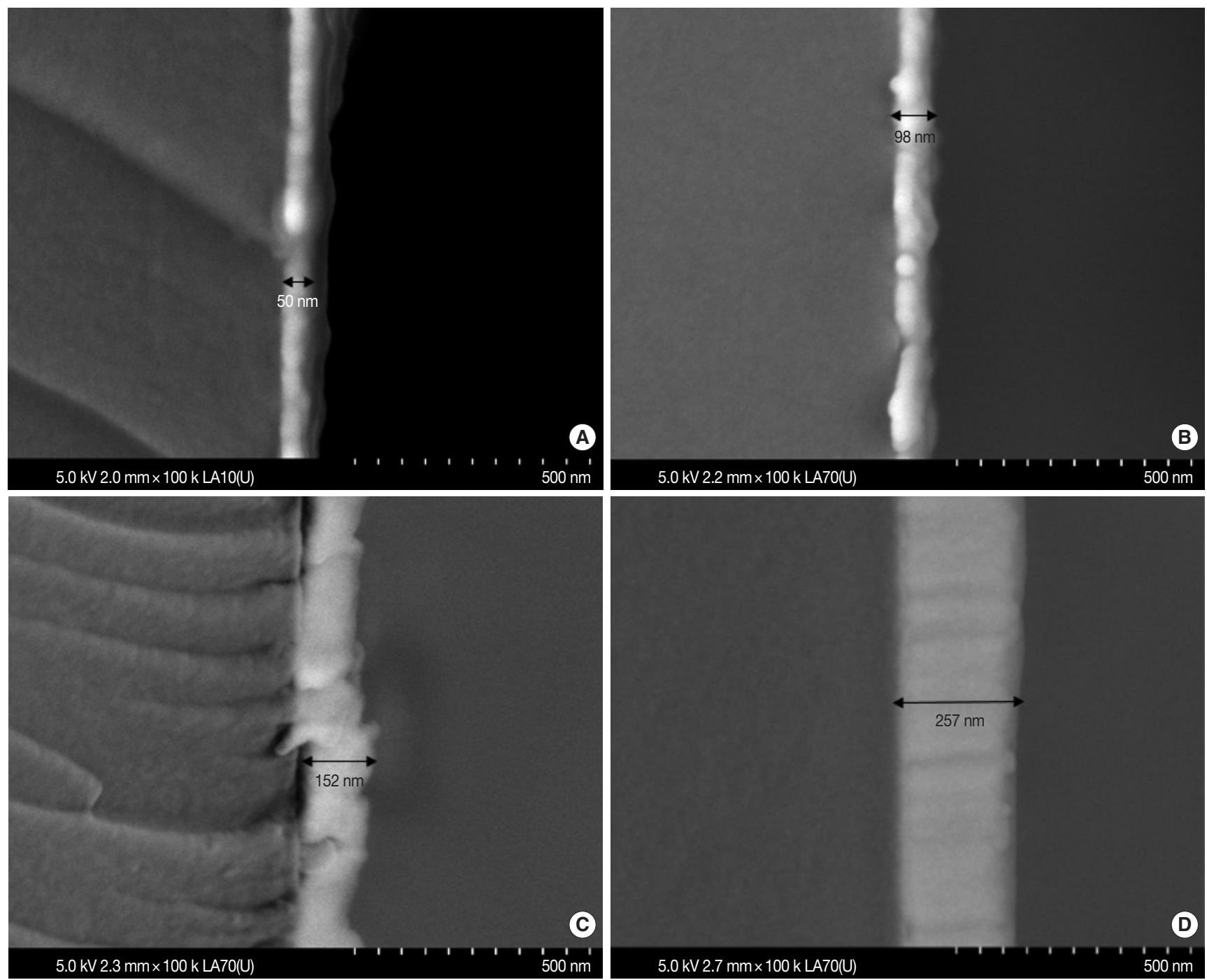

Fig. 2. SEM images of cross-sectional thickness of (A) 20-, (B) 40-, (C) 60-, and (D) 80-second deposited thin-film Ag electrodes on the glass substrate. 


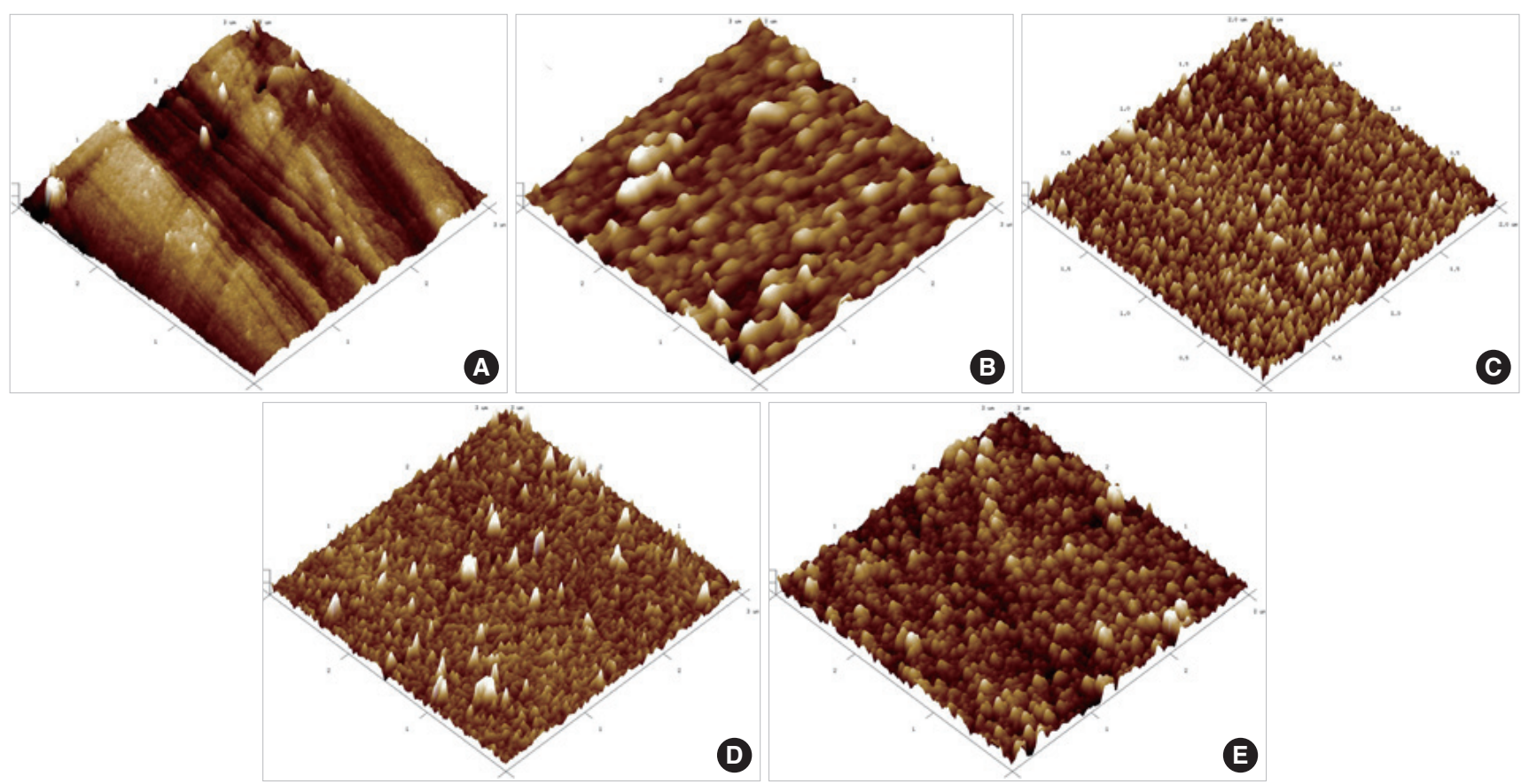

Fig. 3. 3D AFM images of (A) the surface of the bare diamond substrate, and the (B) 20-, (C) 40-, (D) 60-, and (E) 80-second deposited thinfilm Ag electrodes on the glass substrate.

shown in the SEM images of Figure 2 are averaged values.

Figure 3 shows the surface roughness of the deposited thin-film Ag electrodes according to the deposition time. The surface roughness values $(\mathrm{Rq})$ for the bare CVD diamond substrate with 20-, 40-, 60-, and 80-second deposited thinfilm Ag electrodes were $0.69,1.78,2.63,4.55$, and $1.63 \mathrm{~nm}$, respectively. These results demonstrate that the surface roughness of the bare diamond substrate was low, and that a thicker Ag electrode corresponded to increased surface roughness. By comparing the three-dimensional (3D) AFM images, we observed that a 40-second deposition of the thin-film Ag electrode resulted in uniform deposition, i.e., constant surface roughness. However, for the 80 -second deposited film, it was observed that the roughness value of the electrode decreased with increased film thickness.

For the 20-second deposited film, the leakage current was $0.09 \mathrm{nA}$, and the photo-current was an unstable measurement of $39.85 \mathrm{nA}$, as is shown in Figure 4A. It was confirmed that the leakage current was small and the photo-current was large but unstable because of insufficient electrode thickness. When the deposition time was 40 seconds, the leakage current was $0.04 \mathrm{nA}$, and the photo-current was linearly detected as $27.77 \mathrm{nA}$, as is illustrated in Figure 4B. For an approximately $98-\mathrm{nm}$ thick electrode, it was confirmed that leakage current was small; moreover, the thickness of the film resulted in a stable photo-current that was generated by the $\mathrm{UV}$ radiation. The 60 -second deposited film resulted in a leakage current of $0.22 \mathrm{nA}$ and a photo-current that was detected as $26.28 \mathrm{nA}$, as is shown in Figure 4C. When the deposition time was 80 seconds, the leakage current was $0.26 \mathrm{nA}$, and the photo-current was detected as $16.94 \mathrm{nA}$ (Figure 4D).

The results are summarized in Table 1, which shows that the leakage current increased and the photo-current output signal was reduced as the deposition time and thickness of the thin-film Ag electrode increased.

The study results show that the deposition time of a thinfilm Ag electrode strongly influences the surface conditions of the electrode, which thus affect the current characteristics of a radiation detector under UV irradiation. Additionally, it was found that, if the surface conditions of the UV-irradiated CVD diamond detector are not optimal, the detection signal is deteriorated. Thus, through analysis of the results, we have confirmed that thicker deposition and surface uniformity of the thin-film Ag electrode positively influence the leakage current and photo-current of a CVD radiation detector because it facilitates the excellent mobility of holes and electrons.

As the thickness of the thin-film Ag electrode was reduced, the leakage current was observed to decrease, while the detected photo-current signal output became unstable. Conversely, an increase in electrode film thickness was observed 

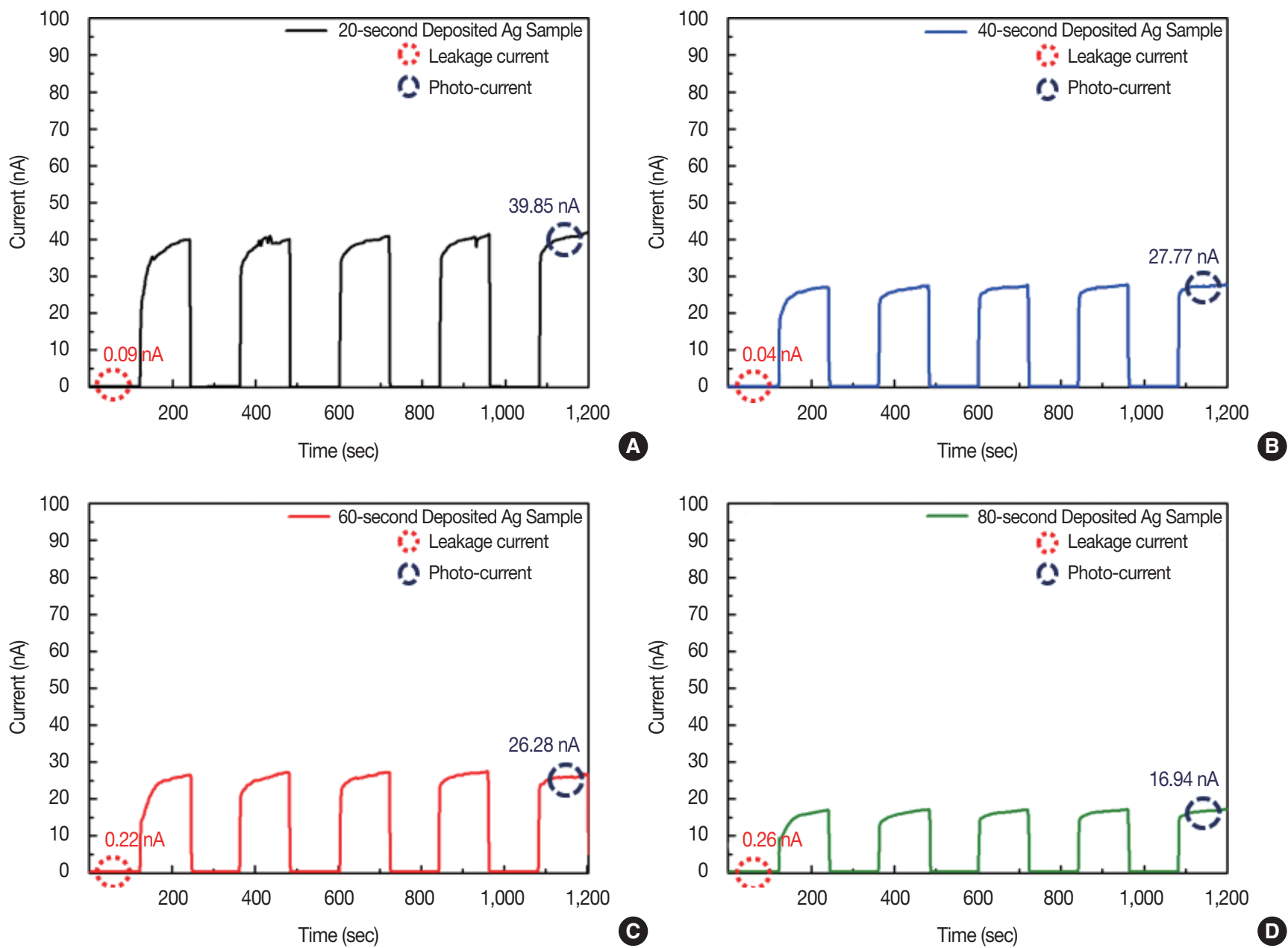

Fig. 4. Leakage current and photo-current under 254-nm UV irradiation for (A) 20-, (B) 40-, (C) 60-, and (D) 80-second deposited thin-film Ag electrodes on CVD diamond.

Table 1. Analysis Results for the Deposited Thin-Film Ag Electrode

\begin{tabular}{lcccc}
\hline $\begin{array}{c}\text { Ag deposition } \\
\text { time (sec) }\end{array}$ & $\begin{array}{c}\text { Leakage } \\
\text { current } \\
\text { (nA) }\end{array}$ & $\begin{array}{c}\text { Photo } \\
\text { current } \\
\text { (nA) }\end{array}$ & $\begin{array}{c}\text { Deposited Ag } \\
\text { cross section } \\
\text { thickness (nm) }\end{array}$ & $\begin{array}{c}\text { Deposited } \\
\text { Ag Rq } \\
\text { (Roughness/nm) }\end{array}$ \\
\hline 20 & 0.09 & 39.85 & $\fallingdotseq 50$ & 1.78 \\
40 & 0.04 & 27.77 & $\fallingdotseq 98$ & 2.63 \\
60 & 0.22 & 26.28 & $\fallingdotseq 152$ & 4.55 \\
80 & 0.26 & 16.94 & $\fallingdotseq 257$ & 1.63 \\
\hline
\end{tabular}

to correspond to increased leakage current and decreased output of the photo-current signal. Additionally, the leakage current was found to be very low, and the photo-current output signal was observed as stable for a deposited film thickness of $98 \mathrm{~nm}$; at this thickness, a uniform and constant surface roughness of the deposited thin-film Ag electrode helped to improve the current characteristics of the CVD diamond radiation detector. These results are in agreement with previous reports that state that high electrical conductivity and low resistivity occur when the thickness of the deposited thin-film Ag is approximately $100 \mathrm{~nm}$ [13].

\section{Conclusion}

Through this study, it was found that the thickness of the thin-film Ag electrode implemented in a radiation detector significantly influences the output signal of the photo-current. Consequently, we have determined the optimal conditions for thin-film Ag electrodes purposed for radiation detectors. We plan to use the findings presented here to develop a stable and efficient detector that can be used in a nuclear reactor.

\section{Acknowledgments}

This work was supported by the National Research Foundation Grant (NRF-2013M2A8A1035822) from Ministry of 
Science and ICT (MSIT) of Republic of Korea.

\section{References}

1. Konorova EA, Kozlov SF, Vavilov VS. Ionization currents in diamonds during irradiation with 500 to 1,000-kev electrons. Soviet Phys.-Solid State. 1966;8:1-5.

2. Almaviva $\mathrm{S}$, et al. Thermal and fast neutron detection in chemical vapor deposition single-crystal diamond detectors. J. Appl. Phys. 2008;103:054501 1-6.

3. Weilhammer P, et al. Recent results on CVD diamond radiation sensors. Nucl. Instrum. Methods Phys. Res., Sect. A. 1998;409(13):264-270.

4. Almaviva S, Marinelli M, Milani E, Prestopino G, Tucciarone A, Verona C, Verona-Rinati G, Angelone M, Pillon M. Extreme UV photodetectors based on CVD single crystal diamond in a p-type/ intrinsic/metal configuration. Diamond Relat. Mater. 2009;18(1): 101-105.

5. Barberini L, Cadeddu S, Caria M, Murgia F. Tests on far UV irradiation of CVD diamond. Nucl. Ins. and Meth. in Phys. Research A. 2000;442(1-3):400-403.

6. Minglong Z, Yiben X, Linjun W, Hujiang S. Response of chemical vapor deposition diamond detectors to X-ray. Solid State Commun. 2004;130(6):425-428.

7. Bergonzo P, Tromson D, Mer C. Radiation detection devices made from CVD diamond. Semicond. Sci. Technol. 2003;18(3): S105-S112.

8. McKeag RD, Jackman RB. Diamond UV photodetectors: sensitivity and speed for visible blind applications. Diamond Relat. Mater. 1998;7(2-5):513-518.

9. Marco Marinelli, et al. Synthetic single crystal diamond as a fission reactor neutron flux monitor. Appl. Phys. Lett. 2007;90:183509.
10. Venkatesan V, Malta DM, Das K, Belu AM. Evaluation of ohmic contacts formed by B+ implantation and Ti-Au metallization on diamond. J. Appl. Phys. 1993;74:1179.

11. Galbiati A, et al. Performance of monocrystalline diamond radiation detectors fabricated using $\mathrm{TiW}, \mathrm{Cr} / \mathrm{Au}$ and a novel ohmic DLC/Pt/Au electrical contact. IEEE Trans. Nucl. Sci. 2009;56(4): 1863-1874.

12. Hauder M, Gstottner J, Hansch W, Schmitt-Landsiedel D. Scaling properties and electromigration resistance of sputtered $\mathrm{Ag}$ metallization lines. Appl. Phys. Lett. 2001;78:838.

13. Gao L, Harter P, Linsmeier C, Wiltner A, Emling R, SchmittLandsiedel D. Silver metal organic chemical vapor deposition for advanced silver metallization. Microelectron. Eng. 2005;82 (3-4):296-300

14. Abdel-Rahman M, Lohstroh A, Sellin PJ. The effect of annealing on the X-ray induced photocurrent characteristics of CVD diamond radiation detectors with different electrical contacts. Phys. Status Solidi A. 2011;208(9):2079-2086.

15. Iwakaji Y, Kanasugi M, Maida O, Ito T. Characterization of diamond ultraviolet detectors fabricated with high-quality singlecrystalline chemical vapor deposition films. Appl. Phys. Lett. 2009;94:223511.

16. Ciancaglioni I, Marinelli M, Milani E, Prestopino G, Verona C, Verona-Rinati G, Angelone M, Pillon M. Secondary electron emission in extreme-UV detectors: Application to diamond based devices. J. Appl. Phys. 2011;110:014501.

17. Pace E, De Sio A. Diamond detectors for space applications. Nucl. Instrum. Methods Phys. Res., Sect. A. 2003;514(1-3):93-99.

18. Periale L, Bizzaro S, Gervino G, Lamarina AM, Palmisano C, Periale R, Picchi P. CVD diamond sensor for UV-photon detection. Nucl. Instrum. Methods Phys. Res., Sect. A. 2012;695:276278. 